\title{
POSTER
}

\section{Dysplasie osseuse floride associée à une fistule cutanée : à propos de deux cas}

\section{Belmehdi A, El Harti K, Benjelloun L, El Wady W}

Service de chirurgie orale, Faculté de médecine dentaire, Université Mohamed V, Rabat, Maroc

\section{Introduction}

Les dysplasies osseuses sont des lésions fibro-osseuses bénignes des maxillaires, développées aux dépens de l'architecture normale de l'os. Ces dysplasies osseuses se présentent sous trois formes, selon leur extension et leur aspect radiologique : périapicale, floride et focale (1).

La dysplasie osseuse floride (DOF) touche essentiellement les femmes d'âge moyen et de race noire. Son diagnostic est clinique et radiologique. Elle se caractérise par de multiples masses sclérotiques des maxillaires le plus souvent bilatérales et symétriques (2).

\section{Observations cliniques}

La première patiente, de race blanche et âgée de 65 ans et la seconde, de race noire et âgée de 70 ans, avaient consulté pour une tuméfaction avec persistance d'une fistule cutanée au niveau de la région submandibulaire gauche. L'orthopantomogramme a montré la présence de radioopacités amorphes et irrégulières au niveau de la mandibule. Le diagnostic, basé sur les résultats cliniques, radiographiques et histopathologiques, a conclu à une DOF.

\section{Discussion}

La DOF est une lésion fibro-osseuse bénigne et rare. Elle a été signalée pour la première fois par Bhaskar et Cutright en 1968 sous le terme d'énostoses multiples (1). Ces auteurs furent les premiers à décrire les critères cliniques, pathologiques et radiologiques de la lésion et à utiliser le terme de dysplasie osseuse floride. La DOF siège uniquement au niveau des maxillaires. Elle n'est associée à aucune autre atteinte squelettique ou anomalie de la formule sanguine. Elle touche les femmes africaines noires d'âge moyen ou avancé et leur descendance. Des cas familiaux ont été retrouvés, la transmission serait autosomique dominante. La DOF est le plus souvent asymptomatique, révélée fortuitement lors d'un examen radiologique. Elle se manifeste parfois par une douleur, un exsudat purulent et une fistule muqueuse et/ou cervicofaciale. Cette infection est due à une exposition endobuccale des masses osseuses. Le symptôme révélateur est rarement une dysmorphie faciale $(2,3)$.

\section{Conclusion}

La dysplasie osseuse floride fait partie du spectre des lésions fibro-osseuses. Elle touche généralement les femmes de race noire et évolue lentement sans signes cliniques. La découverte 
est souvent fortuite suite à un examen radiologique de routine. Le diagnostic est surtout clinique et radiologique. La biopsie est contre-indiquée vu la propension à l'infection. En l'absence de complications infectieuses, la conduite à tenir est l'abstention et la surveillance.

\section{Références}

1-Benazzou L et al. Dysplasie cémento-osseuse floride des maxillaires, Rev Stomatol Chir Maxillofac 2011;112:174-176. 2-Abramovic Ket al. Benign Fibro-Osseous Lesions of the Jaws, Dent Clin N Am 60 (2016) 167-193.

3-Benjelloun L et al. Florid osseous dysplasia: Report of two cases and a review of the literature, Int. J. Odontostomat., 5(3):257-266, 2011. 